\title{
Enhanced Nicotinic Receptor Function and Drug Abuse Vulnerability
}

\author{
Zara M. Fagen, ${ }^{1}$ Robert Mitchum, ${ }^{1}$ Paul Vezina, ${ }^{1,2}$ and Daniel S. McGehee ${ }^{1,3}$ \\ ${ }^{1}$ Committee on Neurobiology and Departments of ${ }^{2}$ Psychiatry and ${ }^{3}$ Anesthesia and Critical Care, University of Chicago, Chicago, Illinois 60637
}

In animals and humans, vulnerability to drug abuse varies among individuals. Animals that display high activity levels in a novel environment are more likely to self-administer psychostimulant drugs, including nicotine, cocaine, amphetamine, and morphine. Recent reports from behavioral studies indicate that nicotinic acetylcholine receptor (nAChR) activity contributes to the rewarding effects of several different addictive drugs. Thus, we hypothesized that differences in nAChR activity may contribute to the predisposition to drug self-administration. After screening of adult rats $(>60 \mathrm{~d}$ postnatal) for the behavioral response to a novel environment, electrophysiological measures of nAChR function were conducted in brain slices that included the mesoaccumbens dopamine neurons of the ventral tegmental area (VTA). We found a positive correlation between the response to novelty and nAChR function in each assay conducted, including nAChR modulation of glutamatergic and GABAergic synaptic inputs to VTA dopamine neurons, as well as somatic nAChR responses of VTA neurons. The response to novelty and sensitivity to addictive drugs are positively correlated with the hormonal response to stress. Consistent with this observation, we found enhanced $\mathrm{nAChR}$ responses in vitro after a $48 \mathrm{~h}$ corticosterone treatment and in vivo after $48 \mathrm{~h}$ of repeated stress. Each of these effects was inhibited by RU486 (11 $\beta$-[ $p$-(dimethylamino)phenyl]-17 $\beta$-hydroxy17-(1-propynyl)estra-4,9-dien-3-one) pretreatment, suggesting a steroid hormone receptor-dependent process. These findings suggest that differences in $\mathrm{nAChR}$ function within the mesoaccumbens dopamine system may contribute to individual differences in drug abuse vulnerability and that these are likely attributable to differences in stress hormone levels.

Key words: nicotinic acetylcholine receptor; glucocorticoid; stress; corticosterone; drug abuse; addiction; acetylcholine; dopamine

\section{Introduction}

Epidemiological data suggest a wide variation in predisposition to drug use and abuse among individuals. In rats, differences in drug self-administration behavior are tightly correlated with the animal's response to a novel environment (Piazza et al., 1998). That is, the high responder (HR) animals that explore a novel environment more actively will acquire drug self-administration with lower drug concentrations, at a faster rate, and to a greater extent than their low responder (LR) peers. These differences between HR and LR rats have been reported for many drugs of abuse, including cocaine, amphetamine, and nicotine (Piazza et al., 1989; Hooks et al., 1991; Suto et al., 2001), and may reflect a common mechanism underlying drug abuse vulnerability. The enhanced self-administration behavior in HR animals is similar to that seen after repeated psychostimulant treatment and has been hypothesized to reflect sensitization of the ascending midbrain dopamine pathways (Piazza et al., 1989; Marinelli and Piazza, 2002). Given that sensitization of these pathways parallels

Received May 11, 2006; revised June 20, 2007; accepted June 28, 2007.

This work was supported by National Science Foundation Grant DGE0202337 (Z.M.F.), National Institutes of Health Grants DA015918 (D.S.M.) and DA09397 (P.V.), and the Brain Research Foundation. We thank Drs. A. Heller and L. Won for the use of MN9D cells, Dr. A. Grace for advice on preparing adult midbrain slices, Dr. M. Marinelli for assistance with the behavioral screen, M. Madonia and L. Baker for technical assistance, and Lindy Barrett for assistance with back labeling and imaging of VTA neurons.

Correspondence should be addressed to Daniel S. McGehee, University of Chicago, Department of Anesthesia and Critical Care, 5841 South Maryland Avenue, MC 4028, Chicago, IL 60637. E-mail: dmcgehee@midway.uchicago.edu. DOI:10.1523/JNEUROSCI.2017-06.2007

Copyright $\odot 2007$ Society for Neuroscience $\quad$ 0270-6474/07/278771-08\$15.00/0 enhanced drug self-administration and seeking (De Vries et al., 1998; Vezina et al., 2002), investigation of brain reward pathways in HR animals may offer insight into the mechanisms associated with inherent predisposition to drug abuse and with increased drug abuse vulnerability in sensitized animals. Furthermore, stress hormones have been implicated as an underlying cause for the HR/LR differences in drug sensitivity (Deroche et al., 1997; Marinelli and Piazza, 2002), and so this model may provide insight to experiential determinants of drug abuse.

Although drugs of abuse target several brain areas, enhanced dopamine transmission from the ventral tegmental area (VTA) to the nucleus accumbens (NAcc) is a key element in the reward (Koob and Le Moal, 1997; Dani, 2003). Dopamine neuron output depends on their intrinsic excitability in combination with afferent regulation by excitatory and inhibitory inputs. Activation of nicotinic acetylcholine receptors (nAChRs) in the VTA excites dopamine neurons via somatic receptors and by modulating both excitatory and inhibitory inputs (Pidoplichko et al., 1997; Mansvelder and McGehee, 2000; Klink et al., 2001; Mansvelder et al., 2002). The high levels of nAChR expression in the VTA, along with the strong endogenous cholinergic inputs from brainstem nuclei, suggest that the nicotinic cholinergic system is an important regulator of dopamine neuron activity. Indeed, dopamine release in the NAcc is modulated by cholinergic activation of the VTA from brainstem cholinergic nuclei (Blaha et al., 1996). Behavioral studies show nAChR involvement in druginduced behavioral states, such as sensitization (Schoffelmeer et 
al., 2002). Interestingly, in addition to mediating the reinforcing properties of nicotine, nAChR activity also contributes to the behavioral effects of psychostimulants, ethanol, and intracranial self-stimulation (Yeomans and Baptista, 1997; Picciotto et al., 1998; Soderpalm et al., 2000; Zachariou et al., 2001; Schoffelmeer et al., 2002). The convergence of evidence implicating nAChRs in reward led us to hypothesize that higher $\mathrm{nAChR}$ sensitivity in $\mathrm{HR}$ versus LR rats may contribute to the differences in drug-induced reward seen behaviorally in these animals. Finally, we predict that differences in stress hormone exposure may contribute to differences in $\mathrm{nAChR}$ function between HR and LR.

\section{Materials and Methods}

Locomotor assay. Harlan Sprague Dawley male rats (180-220 g; Harlan Sprague Dawley, Indianapolis, IN) were singly housed on a reverse light/ dark cycle (lights on from 8:00 P.M. to 8:00 A.M.). Animals were acclimated for $10 \mathrm{~d}$ before testing. To assess the rats' response to novelty, $2 \mathrm{~h}$ of locomotor activity counts were obtained in test chambers to which the rats had no previous exposure. The rats in the upper and lower quartiles for each trial of 12 animals were designated as HR and LR, respectively. All data are expressed as a "locomotor index," in which the animal's locomotor counts were normalized to the average locomotor score obtained during their trial. Rats were continuously housed with minimal disturbance for $\sim 1-14 \mathrm{~d}$ before recording.

Slice preparation. All chemicals were obtained from Sigma (St. Louis, $\mathrm{MO}$ ), unless otherwise specified. Horizontal brain slices from animals were made at the level of the VTA. Animals were anesthetized by isofluorane and rapidly decapitated, and the brain was removed to ice-cold sucrose-artificial CSF (aCSF) with $10 \mu \mathrm{M}$ ascorbic acid [in mM: 252 sucrose, $2.5 \mathrm{KCl}, 1 \mathrm{MgCl}_{2}, 2.5 \mathrm{CaCl}_{2}, 20$ glucose, $25 \mathrm{NaHCO}_{3}, \mathrm{pH} 7.3$ (bubbled continuously with $95 \% \mathrm{O}_{2} / 5 \% \mathrm{CO}_{2}$ )]. Three to four $250 \mu \mathrm{m}$ slices were made in this ice-cold solution and transferred to a $32^{\circ} \mathrm{C}$ holding chamber with normal aCSF ( $125 \mathrm{~mm} \mathrm{NaCl}$ replaced the sucrose in sucrose-aCSF) containing $1 \mathrm{~mm}$ ascorbic acid. The bath was constantly recirculated at $\sim 20 \mathrm{ml} / \mathrm{min}$ until recording.

Back labeling of VTA. To fluorescently label VTA-NAcc projections, rats were anesthetized with intraperitoneal injections of $100 \mathrm{mg} / \mathrm{kg}$ ketamine and $10 \mathrm{mg} / \mathrm{kg}$ xylazine. Bilateral stereotaxic NAcc injections of $0.25 \mu \mathrm{l}$ of Fluoro-red dye (Dong et al., 1996) were performed using the following bregma coordinates: anteroposterior, +3.4 ; lateral, +2.8 ; dorsoventral, -8.1 , angle $10^{\circ}$ off the vertical (Paxinos and Watson, 1998). The animals are allowed to recover for a minimum of $96 \mathrm{~h}$ before anatomical investigation.

Tissue preparation for histology involved induction of deep anesthesia with isoflurane followed by perfusion with $4 \%$ paraformaldehyde and $15 \%$ saturated picric acid in $0.2 \mathrm{M}$ phosphate buffer via the ascending aorta. Brains were removed and immersed in fix for $2 \mathrm{~h}$ and then cryoprotected in $30 \%$ sucrose for $72 \mathrm{~h}$. Sixty-micrometer-thick horizontal sections were made with a cryostat. The NAcc slices were immediately slide mounted for verification of injection sites with anti-fade mountant.

Tissue culture. Clonal mesencephalic cells (MN9D) derived from somatic fusion of embryonic dopaminergic midbrain neurons and neuroblastoma cells were maintained as described previously (Choi et al., 1991). Cells were differentiated in culture with $1 \mathrm{~mm}$ butyric acid for $8 \mathrm{~d}$ before and during recording. For experiments on chronic corticosterone (CORT) exposure, cells were transferred to a steroid-free version of their standard media (DMEM lacking phenol red with charcoal-stripped serum) containing butyric acid at day 4 and treated with $1 \mu \mathrm{M}$ CORT on days 5-7. In some cases, cells were exposed to the glucocorticoid receptor antagonist RU486 (11 $\beta$-[p-(dimethylamino)phenyl]-17 $\beta$-hydroxy-17(1-propynyl)estra-4,9-dien-3-one) (10 $\mu \mathrm{M}$; Steraloids, Newport, RI). As a result, cells received one of three treatments, steroid-free media alone, steroid-free media with $1 \mu \mathrm{M}$ CORT, or steroid-free media with a combination of both CORT and RU486. In cases in which cells received both RU486 and CORT, RU486 was applied at least $2 \mathrm{~min}$ before CORT application.

Electrophysiology: brain slice. Cells were visualized using infrared illumination on an upright microscope (Zeiss, Oberkochen, Germany). Cells were chosen from within a region between 1 and $2.5 \mathrm{~mm}$ from the ventral surface of the midbrain, between 0 and $1.4 \mathrm{~mm}$ from the midline, and between 0.6 and $1.6 \mathrm{~mm}$ caudal from the third ventricle, a clear landmark in the horizontal slice (Paxinos and Watson, 2004). Dopamine neurons were identified by the presence of a hyperpolarization-induced current $\left(I_{\mathrm{h}}\right)$ (see Fig. $\left.1 C\right)$ and larger mean cell diameters $(>20 \mu \mathrm{m})$. However, recent studies have suggested that nondopamine neurons within the VTA express $I_{\mathrm{h}}$ (Ford et al., 2006; Margolis et al., 2006). Therefore, we contend that the $I_{\mathrm{h}}$-positive cells are enriched in dopamine neurons, but this is not likely a pure population. Data was acquired using an Axopatch 1-C amplifier and Digidata 1200 digitizer with pClamp 8.0 software (Molecular Devices, Palo Alto, CA). Standard whole-cell voltage-clamp recordings were conducted with constant bath perfusion at $\sim 3 \mathrm{ml} / \mathrm{min}$ with normal aCSF at room temperature. For some experiments, pharmacological agents were included in the bath perfusate. Excitatory transmission was recorded using a gluconate internal solution (in mM: $154 \mathrm{~K}$-gluconate, $1 \mathrm{KCl}, 1 \mathrm{EGTA}, 10 \mathrm{HEPES}, 10$ glucose, $5 \mathrm{ATP}$, 0.1 and GTP, pH 7.4 with $\mathrm{KOH}$ ), and the external solution contained 20 $\mu \mathrm{M}$ bicuculline to block $\mathrm{GABA}_{\mathrm{A}}$ receptors. Inhibitory transmission was recorded using a high chloride internal solution (in mM: $78 \mathrm{~K}$-gluconate, $77 \mathrm{KCl}, 1$ EGTA, 10 HEPES, 10 glucose, 5 ATP, and $0.1 \mathrm{GTP}$, pH 7.4 with $\mathrm{KOH}$ ), and the external solution contained $10 \mu \mathrm{M}$ DNQX to block AMPA glutamate receptors. The presence of $I_{\mathrm{h}}$ was assessed at $-50 \mathrm{mV}$ using a series of hyperpolarizing voltage steps. All other recordings used a holding potential of $-70 \mathrm{mV}$, with a series resistance $<25 \mathrm{M} \Omega$. Synaptic transmission was analyzed off-line using Minianalysis (Synaptosoft, Decatur, GA) using amplitude and rise time criteria. All events were visually inspected. Rapid application of acetylcholine used a Picospritzer II (General Valve, Fairfield, NJ) and was conducted in the presence of 10 $\mu \mathrm{M}$ DNQX, $20 \mu \mathrm{M}$ bicuculline, $1 \mu \mathrm{M}$ atropine, $1 \mu \mathrm{M}$ TTX, and $300 \mu \mathrm{M}$ $\mathrm{Cd}^{2+}$. Peak currents were measured in real time using pClamp software (Molecular Devices).

Electrophysiology: dissociated cell culture. Standard whole-cell voltageclamp recordings were conducted with a constant bath perfusion of standard external solution (in mM: $140 \mathrm{NaCl}, 2.5 \mathrm{KCl}, 1 \mathrm{MgCl}_{2}, 2 \mathrm{CaCl}_{2}, 10$ HEPES, and 10 glucose, pH 7.4 with $\mathrm{NaOH}$ ). Recording electrodes were filled with gluconate internal solution. Rapid application of agonists in culture used a piezo-controlled drug application system.

Behavioral stress. After the locomotor assay, LR animals were subjected to $2 \mathrm{~d}$ of forced cold water swim stress consisting of $5 \mathrm{~min}$ swims four times each day in $4-6^{\circ} \mathrm{C}$ water. A single cold water swim has been shown previously to cause increases in AMPA receptor function (Saal et al., 2003). The first swim was administered $\sim 2 \mathrm{~h}$ after the onset of the dark cycle, and subsequent swims were separated by $2 \mathrm{~h}$ ( $\pm 15 \mathrm{~min}$ ). For some animals, intraperitoneal injections of RU486 were administered at least $30 \mathrm{~min}$ before the first swim on both days $(40 \mathrm{mg} / \mathrm{kg}$ on day 1 and 25 $\mathrm{mg} / \mathrm{kg}$ on day 2). RU486 at $40 \mathrm{mg} / \mathrm{kg}$ was chosen based on mixed efficacy of $30 \mathrm{mg} / \mathrm{kg}$, and success was shown with this concentration in blocking the effects of stress (Saal et al., 2003). Given that the half-life of RU486 is $25-28 \mathrm{~h}$, dosing was reduced to $25 \mathrm{mg} / \mathrm{kg}$ on day 2 . Animals were killed, and slices were prepared $\sim 15-18 \mathrm{~h}$ after the last swim.

Statistical analysis. Datasets correlating individual responses with locomotor activity used a linear regression by Systat (SPSS, Chicago, IL) to determine significance. Any outliers identified by the software based on the studentized residual were excluded from the dataset. Student's $t$ tests were used to compare responses between groups, including the response to novelty in Figure 1 and the HR, LR, stressed LR, and stressed plus RU486 groups in Figure 6. In the synaptic modulation experiments, responsive cells were identified by conducting a Student's $t$ test on the mean synaptic current frequency (per $10 \mathrm{~s}$ bin) for 1 min immediately before nicotine application and 1 min centered either on the peak nicotine effect or $1 \mathrm{~min}$ after starting the nicotine application (in the absence of any clear effect). $\chi^{2}$ tests were used to determine the significance of response prevalence between HR and LR groups in Figures 2 and 3. For comparing responses to focal application of ACh across the locomotor range, cell sensitivity was determined by averaging responses to at least two focal applications of $1 \mathrm{~mm}$ ACh onto each cell. To minimize effects of receptor desensitization that might lead to underestimation of the peak current, only those currents with $10-90 \%$ rise times $<100$ ms were used for our analysis. Data collected from multiple cells from each animal were 
averaged for correlation to locomotor score. Small ACh responses that would elicit $<3 \mathrm{mV}$ depolarization, as determined by input resistance, were not included in the correlation analysis.

\section{Results}

In this study, cohorts of 12 male rats (Harlan Sprague Dawley) were screened for their locomotor activity in a novel environment. Locomotor activity was monitored for a $2 \mathrm{~h}$ period, and each animal's locomotor count was normalized to the average for that cohort. Those animals with locomotor counts within the upper and lower quartiles of each cohort were identified as HR and LR (Fig. 1 $A$ ) and were then used for electrophysiological studies. Horizontal brain slices including the VTA (Fig. $1 B$ ) were used for whole-cell voltage-clamp recordings. Back labeling of VTA neurons was performed to illustrate the mesoaccumbens projections by injecting fluorescent tracer into the NAcc shell (Fig. $1 B$ ). VTA recordings were conducted within a region that corresponds to the observed back labeling. This included cells between 1 and $2.5 \mathrm{~mm}$ from the ventral surface of the midbrain, between 0 and $1.4 \mathrm{~mm}$ from the midline, and between 0.6 and 1.6 $\mathrm{mm}$ caudal from the third ventricle, a clear landmark in the horizontal slice (Paxinos and Watson, 2004). In the slice recording experiments, dopamine neurons in this region were identified by the presence of a hyperpolarization-induced current $\left(I_{\mathrm{h}}\right)$ (Fig. $1 C)$ and larger mean cell diameters. However, recent studies have suggested that nondopamine neurons within the VTA express $I_{\mathrm{h}}$ (Ford et al., 2006; Margolis et al., 2006). Therefore, our criteria have identified a population of neurons that is enriched in dopamine neurons but is not likely a pure population.

\section{Stronger nicotinic enhancement of excitatory inputs to VTA dopamine neurons in HR rats}

Whole-cell recording in VTA reveals that nicotine $(1 \mu \mathrm{M})$ enhances excitatory synaptic inputs to a subset of dopamine neurons, as shown by an increase in spontaneous EPSC (sEPSC) frequency (Fig. 2A). The nAChRs that mediate this effect of nicotine are located on presynaptic glutamate terminals and are most likely the $\alpha 7$ subtype, as we found previously in neonatal rat VTA (Mansvelder and McGehee, 2000). Support for the hypothesis of stronger $\mathrm{nAChR}$ responses in HR animals was seen in the higher prevalence of nicotine-induced increases in sEPSC frequency (five of six HR; four of nine LR). In addition to the higher incidence of nicotinic responses, $\mathrm{HR}$ animals had stronger responses to nicotine. Figure $2 B$ illustrates the correlation between the nicotine-induced increase in sEPSC frequency and the normalized locomotor response to novelty (linear regression $r=$ $0.641 ; p<0.05 ; n=15$ neurons from 15 different rats). Therefore, nAChR activation induces more profound increases in excitatory drive to dopamine neurons in HR compared with LR animals. The basal sEPSC frequency did not differ across the range of locomotor responses to novelty (Fig. 2C).

Behaviorally, differences in drug self-administration between HR and LR rats are attributable to a vertical shift in the doseresponse function in predisposed animals (Piazza et al., 2000). In HR animals, addictive drugs induce stronger behavioral responses than in LR rats, including stronger motivation as assessed in self-administration tests (Piazza et al., 1989; Suto et al., 2001).

\section{Stronger nicotinic modulation of inhibitory inputs to VTA dopamine neurons in HR rats}

Inhibitory transmission onto VTA dopamine neurons is transiently increased by bath application of nicotine $(1 \mu \mathrm{M})$ (Fig. $3 A)$. In our previous studies, the nicotine-induced enhancement of
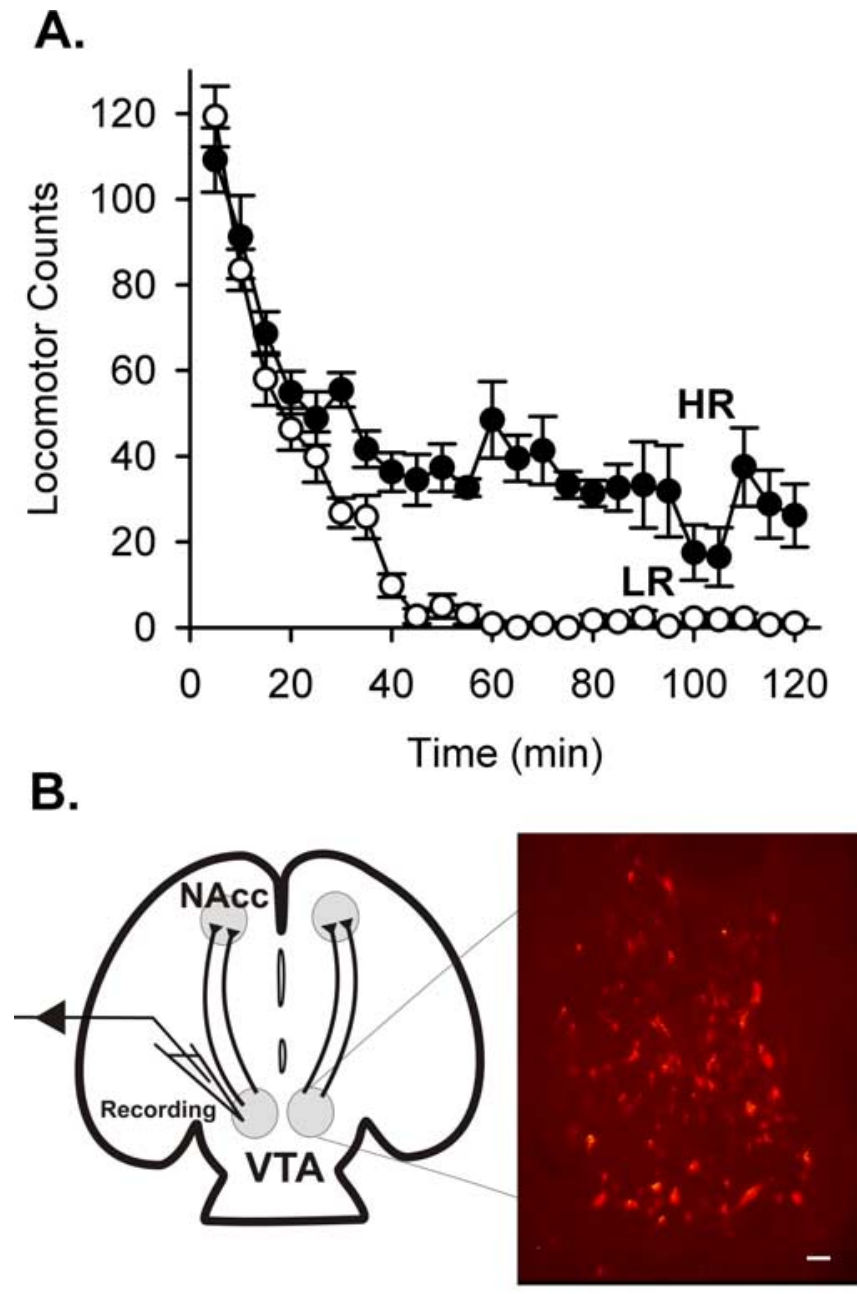

C.

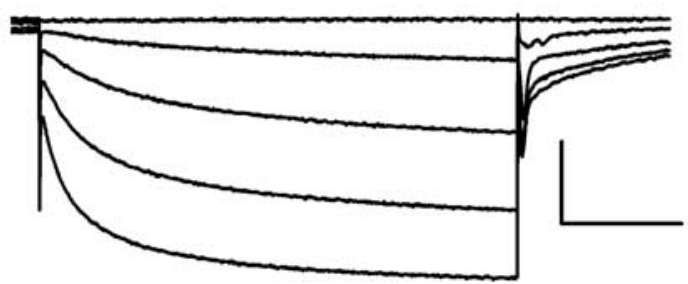

Figure 1. A, Locomotor activity in a novel environment observed in two cohorts of 12 rats. The average activity levels are plotted for the animals from the upper and lower quartiles from each activity screen ( $n=6 \mathrm{HR}$ and $6 \mathrm{LR}$ rats). $\boldsymbol{B}$, Schematic diagram of the horizontal slice preparation, which includes the VTA and its afferent inputs. Right is a photomicrograph of VTA neurons back labeled by injection of Fluoro-red dye into the NAcc. Scale bar, $100 \mu \mathrm{m}$. C, Dopamine neurons were identified based on cell diameter and the presence of an $I_{\mathrm{h}}$ current while holding at $-50 \mathrm{mV}$, in response to hyperpolarizing voltage steps to $-65,-80,-95$, and $-110 \mathrm{mV}$. Neurons with soma diameters $>20 \mu \mathrm{m}$ that expressed $I_{\mathrm{h}}$ currents $>20 \mathrm{pA}$ were considered to be dopaminergic. Calibration: $400 \mathrm{pA}, 1 \mathrm{~s}$.

inhibitory transmission was shown to be dependent on action potential activity in GABA neurons. This suggests that the nAChRs are located some distance from the axon terminal, perhaps primarily in somatodendritic regions (Mansvelder et al., 2002). Desensitization of these receptors by nicotine was found to depress GABAergic input to the dopamine neuron, presumably by removing the influence of tonic cholinergic input to these cells (Garzón et al., 1999; Mansvelder et al., 2002). Consistent with this observation, in recordings in which nicotine enhanced GABAer- 
A.

Control

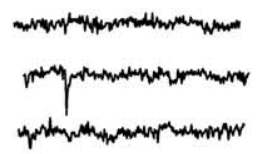

$1 \mu \mathrm{M}$ Nicotine

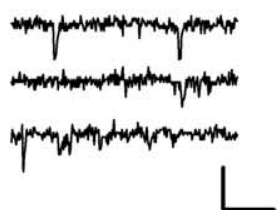

$$
\begin{aligned}
& 0 \\
& 0 \\
& 0 \\
& 0 \\
& \text { o } \\
& 0 \\
& 0 \\
& \text { w } \\
& \text { क }
\end{aligned}
$$

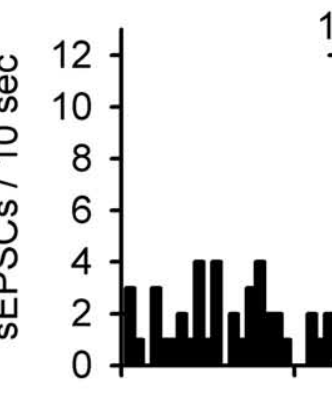

$1 \mu \mathrm{M}$ Nicotine

B.

0

200

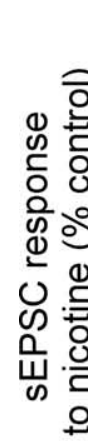

280 Time $(\mathrm{sec})$

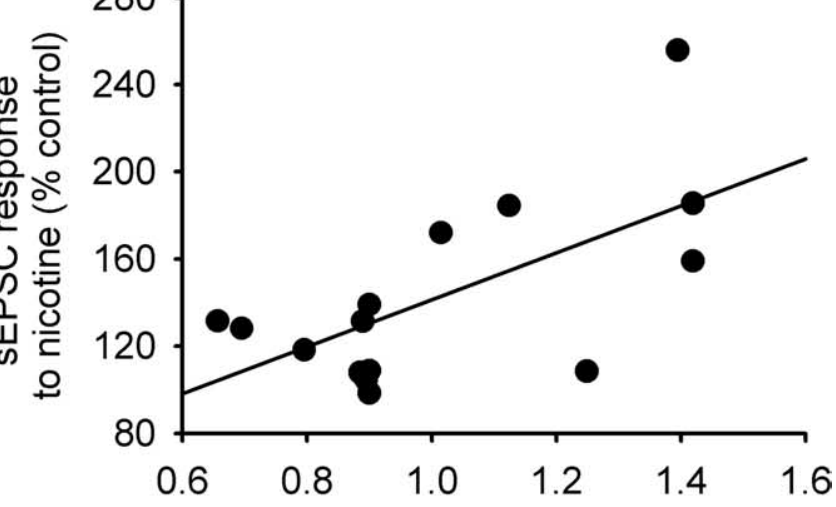

C.

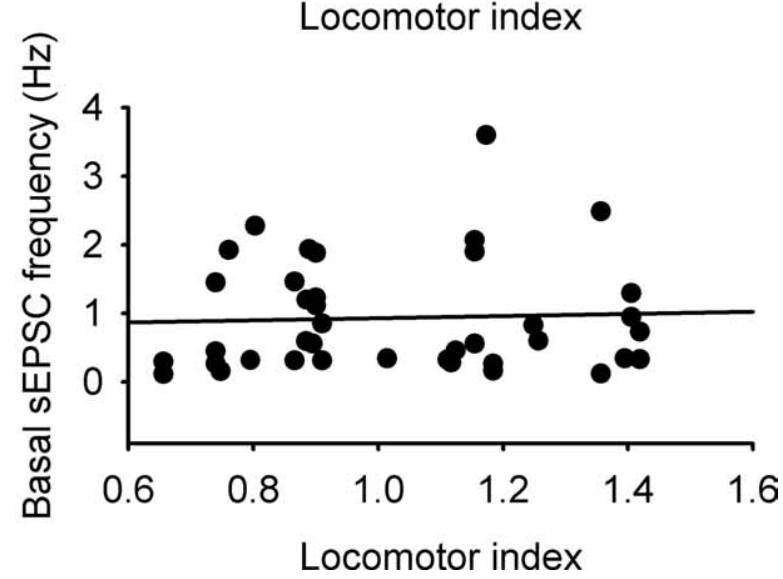

Figure 2. HR rats show enhanced nAChR modulation of spontaneous excitatory input to dopamine neurons of the VTA. Excitatory transmission was monitored in dopamine neurons using whole-cell voltage clamp in the presence of bicuculline $(20 \mu \mathrm{m})$, a selective $G A B A_{A}$ antagonist. All synaptic transmission was recorded at $-70 \mathrm{mV}$. $A$, Sample traces during control and nicotine treatment illustrates the increase in SEPSC frequency with bath application of nicotine $(1 \mu \mathrm{M})$. The sample frequency histogram shows an increase in $\mathrm{SEPSC}$ frequency with bath-applied nicotine $(1 \mu \mathrm{M})$. For this example, the frequency increased $233.9 \%$ of baseline activity $(p<0.05)$. $\boldsymbol{B}$, The magnitude of the response to nicotine is positively correlated with locomotor activity ( $r=0.641 ; p<0.02 ; n=15$ cells from 15 animals). HR animals had a higher fraction of cells that respond to nicotine with an increase in $S E P S C$ frequency relative to $L R$ animals $(p<0.05)$. C, Basal sEPSC frequency did not correlate with locomotor activity.
A.

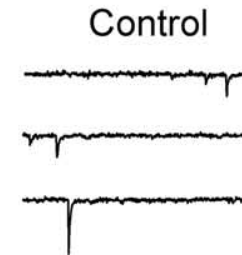

$1 \mu \mathrm{M}$ Nicotine
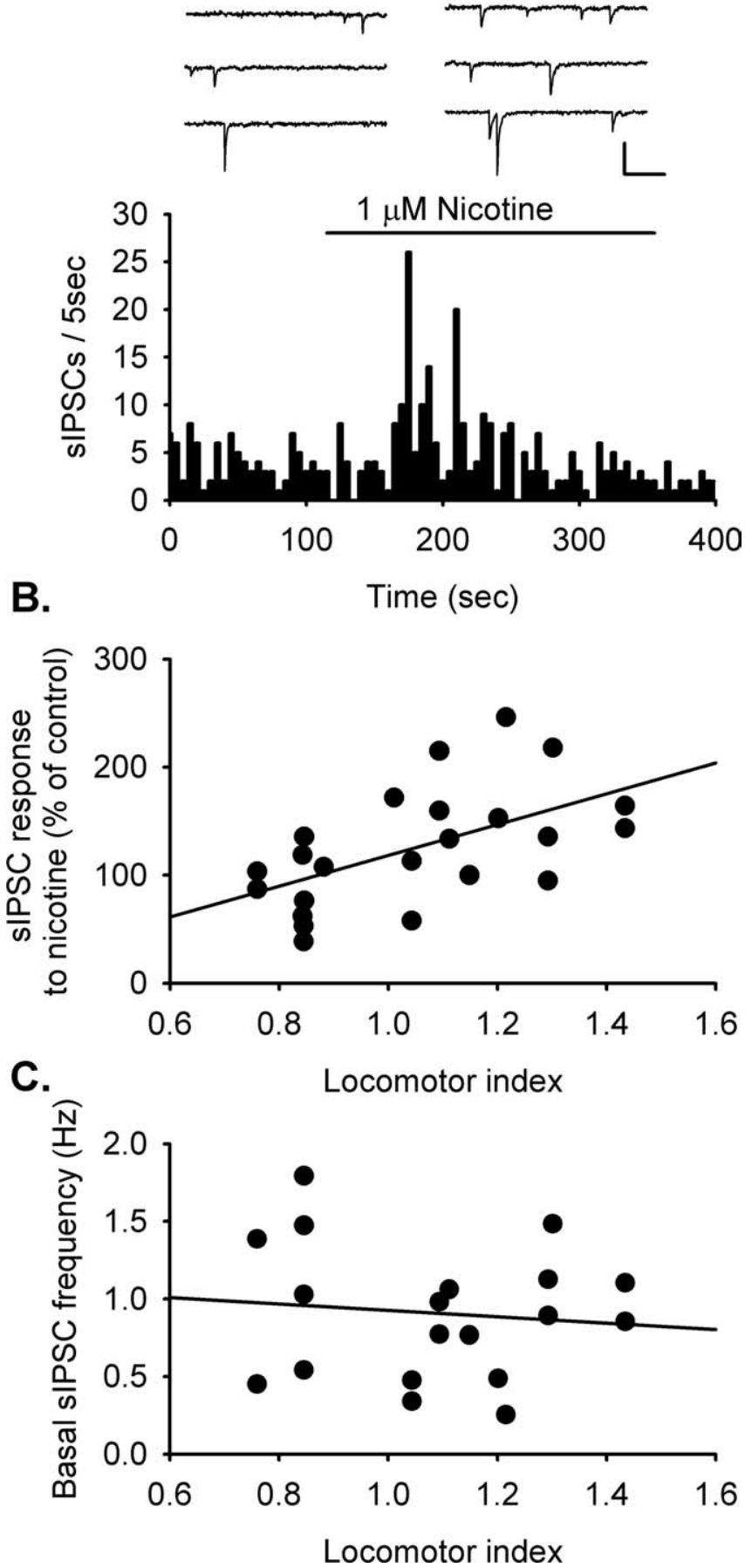

Figure 3. HRrats show enhanced $n A C h R$ modulation of spontaneous inhibitory transmission to VTA dopamine neurons. Inhibitory transmission was monitored in VTA dopamine neurons using whole-cell voltage clamp in the presence of DNQX (10 $\mu \mathrm{M})$ to block AMPA glutamate receptors. All synaptic transmission was recorded at $-70 \mathrm{mV}$. Symmetrical chloride concentrations were used to facilitate analysis, which results in inward currents with $\mathrm{GABA}_{A}$ receptor activation. $\boldsymbol{A}$, Sample traces show SIPSCs during control and in the presence of nicotine $(1 \mu \mathrm{M})$. The sample frequency histogram shows an increase in sIPSC frequency with bath-applied nicotine $(1 \mu \mathrm{M})$. For this example, the sIPSC frequency increased to $215.0 \%$ of baseline $(p<0.05)$. After this transient increase, sIPSC frequency decreased to $64.6 \%$ of baseline $(p<0.05)$. B, The magnitude of the response to nicotine is positively correlated with locomotor activity $(r=$ $0.532 ; p<0.01 ; n=23$ cells from 23 animals). HR animals had a higher fraction of cells that respond to nicotine with an increase in sIPSC frequency relative to LR animals $(p<0.05)$. C, Basal sIPSC frequency did not correlate with locomotor activity. 
gic input to VTA dopamine neurons, $60 \%$ of these also showed a decrease in spontaneous IPSC (sIPSC) frequency below baseline after the transient increase $(n=6$ of 10$)$ (Fig. $3 A$ ).

Additional support for the idea that HR rats have stronger $\mathrm{nAChR}$ responses was seen in the larger fraction of dopamine neurons that responded to nicotine with an increase in sIPSC in HR than LR animals ( 6 of $11 \mathrm{HR}, 4$ of $12 \mathrm{LR}$ ). In addition, the magnitude of the effects of nicotine on sIPSC frequency correlated positively with locomotor activity (linear regression $r=$ 0.532; $p<0.01 ; n=23$ neurons from 23 different rats) (Fig. $3 B$ ). The enhancement of GABA inputs may offset the enhanced glutamate activity described above, but the subsequent decrease in GABA input would ultimately lead to a disinhibition of the dopamine neuron. We did not see a significant difference between HR and LR rats in the magnitude of this disinhibition after nicotine exposure. Our prediction is that stronger nAChR modulation of GABA release in HR animals would result in stronger disinhibition of VTA dopamine neurons after nicotine exposure, but this is dependent on maintained endogenous cholinergic input to the GABA neurons in our slices. The depression of GABAergic input is less prevalent than the enhancement in these and previous studies (Mansvelder et al., 2002), which likely reflects variation in cholinergic activity in our slices. Although our slice preparation includes part of the brainstem cholinergic centers, future experiments using alternate slice orientation will be required to more completely address the question of potential differences between HR and LR rats in the disinhibitory effects of nicotine.

Neither basal inhibitory nor excitatory transmission differed between HR and LR animals (Figs. 2C, 3C), suggesting that differences in the modulation of synaptic transmission is not likely a consequence of differences in the baseline activity of these inputs. Given our evidence that endogenous cholinergic drive affects GABAergic input to dopamine neurons, there may be a compensatory decrease in cholinergic drive in the VTA of HR rats. Unfortunately, direct assessment of nicotinic cholinergic synaptic input to either dopamine or nondopamine neurons in the VTA has not been possible. Regardless of the reason for similar basal inputs, these results demonstrate stronger $\mathrm{nAChR}$ modulation of synaptic transmission and stronger nAChR-mediated excitation of dopamine neurons in HR animals.

\section{Stronger somatic $\mathrm{nAChR}$ responses in VTA of HR rats}

Somatic nAChR expression was assayed through focal application of ACh (1 mM) onto dopamine and nondopamine neurons of the VTA using focal pressure application. Peak currents induced by ACh were normalized to cell capacitance. Only those currents that were determined to elicit $>3 \mathrm{mV}$ depolarization based on input resistance were used for this analysis. Focal application of ACh reliably elicited fast inward currents attributed to nAChR activation (Fig. $4 A$ ).

Somatic nAChR response magnitudes from VTA dopamine neurons were positively correlated with locomotor value from the novelty screen $(r=0.771 ; p<0.05 ; n=14$ neurons from 8 rats) (Fig. $4 B$ ). In addition, putative GABAergic cells of the VTA, which lacked $I_{\mathrm{h}}$ current, also expressed nAChR response magnitudes that positively correlated with locomotor activity $(r=$ 0.674; $p<0.05 ; n=18$ neurons from 10 rats) (Fig. $4 C$ ). The stronger $\mathrm{nAChR}$ responses in HR animals was not merely attributable to an upregulation of all ion channel activity, because $I_{\mathrm{h}}$ magnitude did not differ across the spectrum of the behavioral response to novelty ( $n=58$ neurons) (Fig. $4 D$ ). These data support the idea that differences in somatic nAChR responses in this dopaminergic nucleus may contribute to the observed differ- ences in drug self-administration behavior between HR and LR rats.

\section{Corticosterone enhancement of $\mathrm{nAChR}$ responses}

In vivo studies have shown that variation in stress hormone levels and stress history may underlie the individual differences in motivational effects of a variety of addictive drugs. Exogenous administration of CORT can eliminate differences between HR and LR rats in drug self-administration (Piazza et al., 1991; Mantsch et al., 1998), and HR animals have more prolonged elevation of stress hormones both during their circadian rhythm and in response to stress (Marinelli and Piazza, 2002). The differences in CORT levels between HR and LR rats could induce differences in nAChR function through cytosolic glucocorticoid or mineralocorticoid receptors, which affect gene transcription or via cellsurface receptors (Rupprecht and Holsboer, 1999). Some nAChR subtypes are inhibited by glucocorticoids (Shi et al., 2001), and it has been reported that $\mathrm{nAChRs}$ can undergo upregulation after exposure to steroid antagonists (Almeida et al., 2000). This lead us to hypothesize that $\mathrm{nAChR}$ function may be upregulated in HR animals attributable to chronic nAChR inhibition by CORT.

To test this hypothesis, we determined the effects of acute and chronic CORT exposure in a dopaminergic cell line (MN9D) (Choi et al., 1991). MN9D cells were differentiated with n-butyrate for $7 \mathrm{~d}$ to induce a dopamine neuron-like phenotype (Choi et al., 1991). For acute studies, the differentiated cells were transferred to steroid-free media, and nAChR sensitivity was tested using focal application of nicotine $(100 \mu \mathrm{M})$. In agreement with observations in other studies, 1-2 min pretreatment with CORT induced dose-dependent inhibition of nAChRs with an $\mathrm{IC}_{50}$ of $40.1 \mu \mathrm{M}$ (data not shown). We found a small but significant inhibition at stress-associated CORT concentrations $(1 \mu \mathrm{M})$, which is consistent with observations in other systems showing nAChR inhibition by physiological CORT levels (Shi et al., 2001).

To test the effects of chronic CORT treatment on differentiated MN9D cells, the media was replaced with steroid-free media alone, CORT $(1 \mu \mathrm{M})$, or CORT $(1 \mu \mathrm{M})$ with the glucocorticoid receptor antagonist RU486 $(10 \mu \mathrm{M})$. nAChR currents were measured 1, 24, and $48 \mathrm{~h}$ after the initiation of these treatments, using sequential application of nicotine $(100 \mu \mathrm{M})$ and the selective $\alpha 7$ receptor agonist choline $(3 \mathrm{~mm})$. Peak currents were measured in the absence of CORT and normalized to cell capacitance. Chronic CORT treatment induced a time-dependent increase in $\mathrm{nAChR}$ responses in the MN9D cells $(r=0.479 ; p<0.01 ; n=$ 6-9 for each time point and condition) that was inhibited by RU486 treatment (Fig. 5). The nAChR currents were significantly different from those seen in control cultures at the $48 \mathrm{~h}$ time point, whereas cells treated with both CORT and RU486 showed no difference from age-matched controls. The time required for the CORT-induced change along with the RU486 sensitivity suggest that the upregulation of $\mathrm{nAChR}$ function depends on genomic effects. Furthermore, there was no difference in the ratio of peak currents induced by choline and nicotine after $48 \mathrm{~h}$ CORT treatment compared with age-matched controls (control, $1.3 \pm$ $0.07, n=6$; nicotine, $1.6 \pm 0.1 ; n=8 ; p=0.75$ ). This observation suggests that the effect of CORT is not confined to one nAChR subtype but had comparable effects on both $\alpha 7$ and non- $\alpha 7$ nAChRs. It is unlikely that nAChR upregulation is occurring through direct interaction of the steroid with the receptor protein. Most importantly, these data support the notion that individual differences in CORT levels may contribute to the observed differences in $\mathrm{nAChR}$ sensitivity between HR and LR rats. 

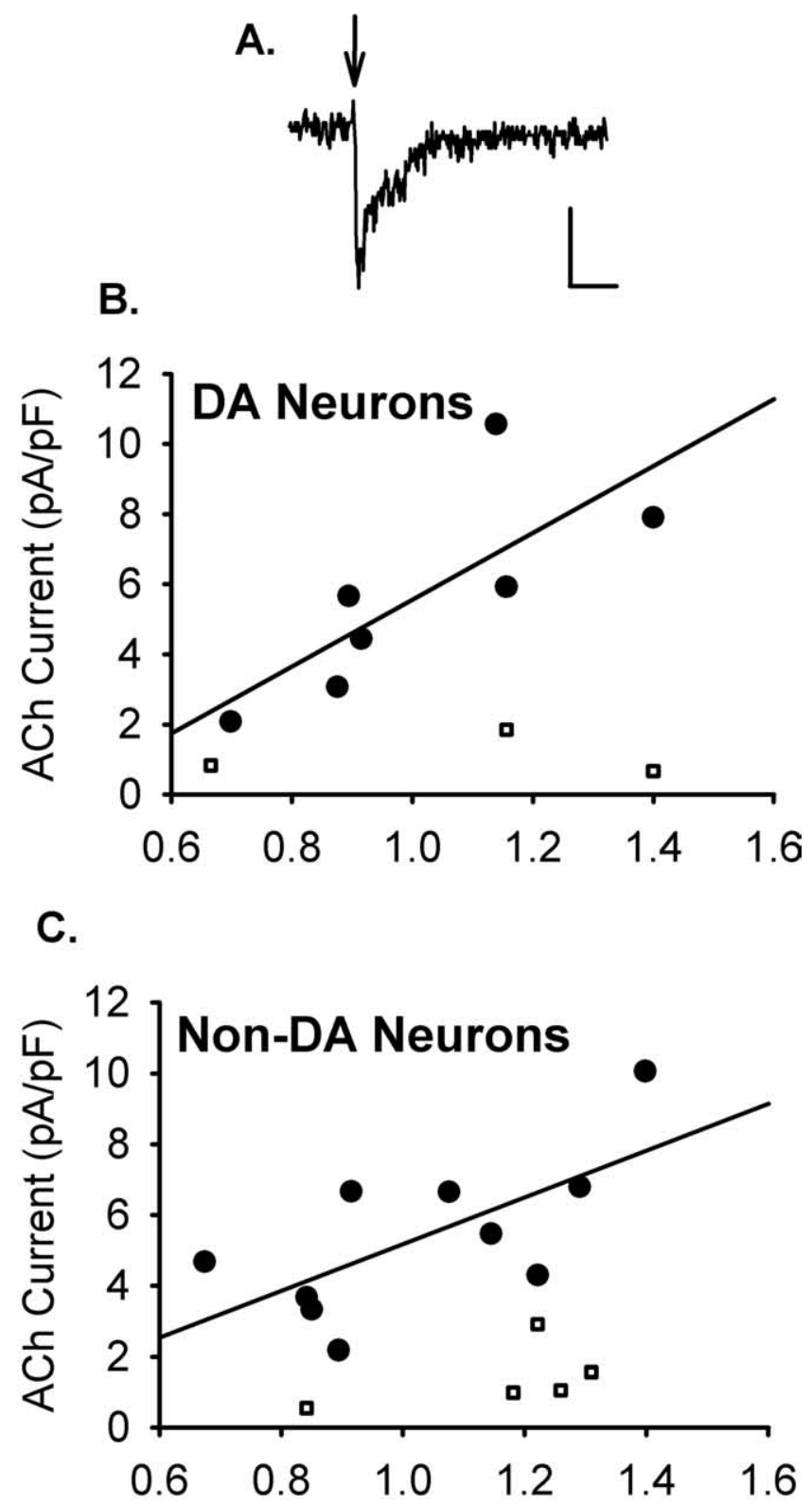

D.

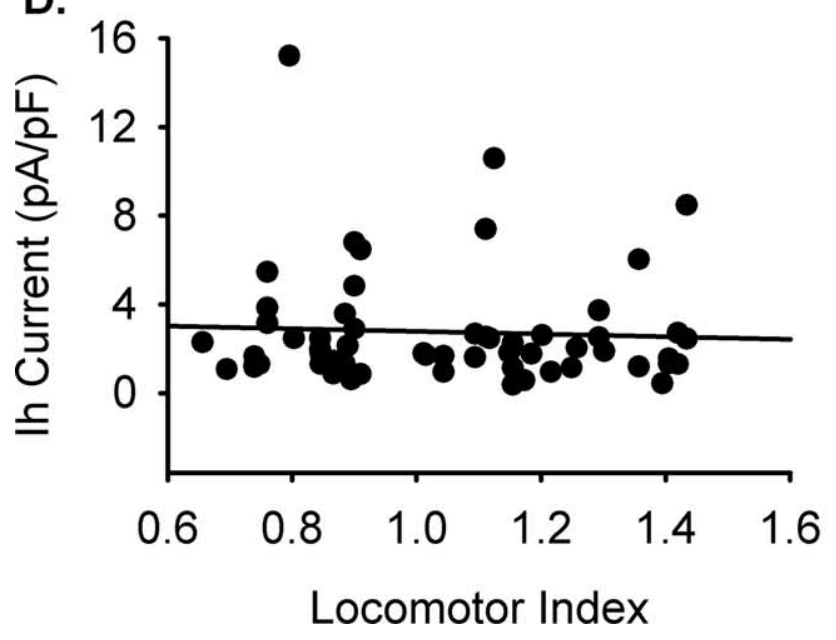

Figure 4. Somatic $n A C h R$ responses in the VTA correlate with locomotor activity. $A$, Example of a current evoked by focal pressure application of $A C h(1 \mathrm{~mm}, 30 \mathrm{~ms})$ to a VTA dopamine neuron in a slice from an HR rat. Calibration: $20 \mathrm{pA}, 1 \mathrm{~s}$. Nicotinic currents were isolated by TTX $(1 \mu \mathrm{m})$,

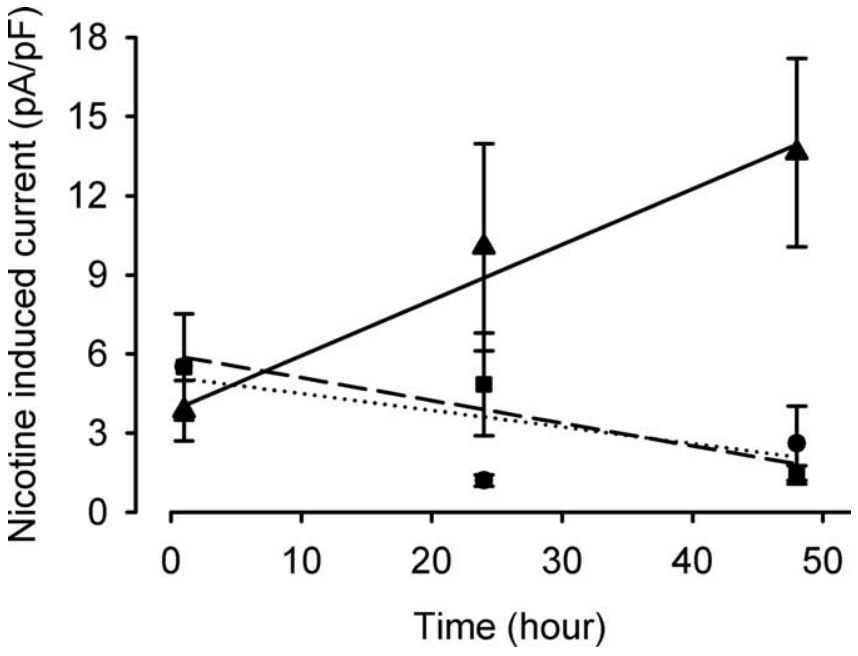

Figure 5. Chronic CORT treatment increases nicotinic receptor function in vitro. MN9D cells were differentiated for $5 \mathrm{~d}$ before treatment with steroid-free media alone $(\mathbf{)})$, CORT $(1 \mu \mathrm{M})$ $(\mathbf{\Delta})$, or CORT $(1 \mu \mathrm{M})$ and RU486 (10 $\mu \mathrm{m})(\mathbf{\square})$ for 1-50 h. Whole-cell voltage-clamp experiments in the absence of extracellular CORT analyzed nAChR function through focal nicotine application $(100 \mu \mathrm{M})$ using a piezo-controlled rapid application system, with $-70 \mathrm{mV}$ holding potential. After chronic CORT treatment, MN9D cells showed a time-dependent increase in nicotine-induced currents $(r=0.479 ; p<0.005)$. Nicotine-induced currents in CORT-treated cells were significantly increased after $2 \mathrm{~d}$ compared with age-matched controls $(p<0.007)$. Inhibition of the glucocorticoid receptor by RU486 blocked this increase.

Stress-induced enhancement of nAChR responses in LR rats To further investigate whether electrophysiological differences in nAChR function in HR and LR animals are related to stress hormone levels, we examined the effects of environmental stress on $\mathrm{nAChR}$ function. LR rats were forced to swim in cold water (4$6^{\circ} \mathrm{C}$ ) for 5 min four times daily at $2 \mathrm{~h}$ intervals for 2 consecutive days. The following day, horizontal brain slices were obtained from the animals, and nicotine-induced modulation of glutamatergic input to VTA dopamine neurons was assayed. For a subset of LR rats, intraperitoneal injections of RU486 were administered at least $30 \mathrm{~min}$ before their first swim on both days $(40 \mathrm{mg} / \mathrm{kg}$ on day $1,25 \mathrm{mg} / \mathrm{kg}$ on day 2).

Chronic stress of LR rats abolished the difference in nAChRmediated modulation of sEPSC frequency between HR and LR animals ( $p>0.56 ; n=8$ animals) (Fig. 6). In agreement with the in vitro studies above, the stress effects on $\mathrm{nAChR}$ function are glucocorticoid receptor dependent. Systemic administration of RU486 eliminated the effects of stress on the nicotine sensitivity of LR rats. The average nicotine-induced change in sEPSC frequency that was not different from nonstressed LR animals but was significantly smaller than those observed in HR and stressed LR rats $(n=6 ; p<0.05)$. When HR rats were subjected to the same stress protocol, we saw no additional increase in nAChR modulation of sEPSC frequency relative to nonstressed HR rats

$\leftarrow$

DNQX (10 $\mu \mathrm{m})$, bicuculline $(20 \mu \mathrm{M}), \mathrm{Cd}^{2+}(300 \mu \mathrm{M})$, and atropine (1 $\left.\mu \mathrm{M}\right)$. B, Dopamine (DA) neurons in the VTA show increased nAChR function in animals with high locomotor values $(r=$ $0.771 ; p<0.05 ; n=14$ responses from 8 rats). $C$, Nondopamine neurons of the VTA showed significantly larger responses in HR animals $(r=0.674 ; p<0.05 ; n=18$ responses from 10 rats). Data presented for both dopamine and nondopamine neurons are averages of the AChinduced currents obtained from each animal (1-3 responses were recorded from each animal). The linear regressions were performed against the responses that elicited $>3 \mathrm{mV}$ depolarization according to input resistance (filled circles). Cell responses that elicited $<3 \mathrm{mV}$ depolarization have been plotted, as well (open squares). $\boldsymbol{D}$, The linear regression of $/ \mathrm{h}$ current magnitude and locomotor index shows no significant correlation $(n=58)$. 


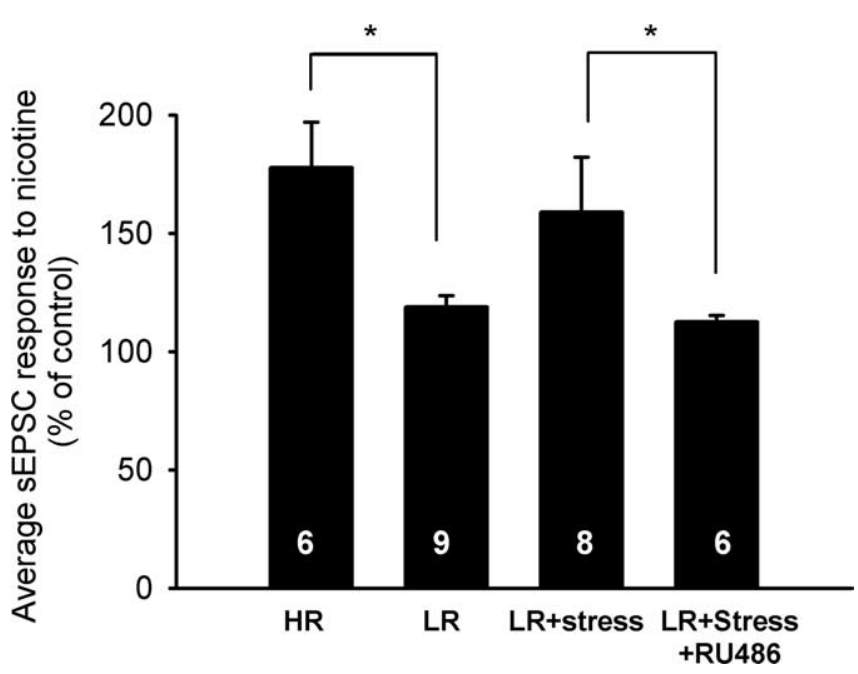

Figure 6. Stressful experiences result in increased $n A C h R$ function in $L R$ animals through a glucocorticoid receptor-dependent mechanism. Animals subjected to a series of cold forced swim tests on 2 consecutive days were then assayed for nAChR modulation of excitatory inputs to VTA dopamine neurons. The magnitude of the increase in SEPSC frequency is significantly higher in HR than nonstressed LR animals ( $p<0.05$; $177.6 \pm 19.5$ and $118.8 \pm 4.8 \%$, respectively). Stressing $L R$ animals led to larger nicotine responses (158.8 $\pm 23.4 \%$ ) and eliminated the difference between HR and LR animals. In addition, RU486 ( 40 and $25 \mathrm{mg} / \mathrm{kg}$ on days 1 and 2, respectively) significantly reduced the effects of stress on nicotinic modulation of sEPSCs $(p<0.05 ; 112.5 \pm 2.8 \%)$. Data are mean \pm SEM; * $p<0.05$.

$(147 \pm 19 \%$ of control frequency; $n=4 ; p>0.6$ for each comparison). We conclude that stress does not enhance nAChR modulation of sEPSC frequency in HR rats. It is likely that the inherently elevated CORT level in HR rats is sufficient to induce the effect. The ability of stress to enhance nAChR function in the VTA of LR animals provides a mechanism by which the reinforcing effects of drugs can be modulated through experience.

\section{Discussion}

These data support a central mechanism for drug abuse vulnerability through alterations in $\mathrm{nAChR}$ function in brain reward areas. Modulation of $\mathrm{nAChR}$ activity within the mesoaccumbens dopamine system may be one mechanism whereby stress hormones affect drug abuse vulnerability. We demonstrated that HR rats have stronger $\mathrm{nAChR}$-mediated effects on synaptic transmission in VTA and stronger direct nAChR responses of both dopaminergic and nondopaminergic VTA neurons. Precisely how these differences contribute to drug sensitivity and predisposition to self-administration will require additional study.

Our working hypothesis is that the net effect of stronger nAChR responses makes VTA neurons, particularly the dopamine neurons, more excitable. Anatomical evidence has shown that cholinergic innervation from brainstem nuclei to the VTA impinges on both dopaminergic and nondopaminergic cell types (Omelchenko and Sesack, 2005, 2006). Stronger somatic effects of nAChRs will contribute to increased excitability directly. In addition, the combination of greater prevalence and magnitude of nAChR-mediated enhancement of glutamatergic input to VTA dopamine neurons will also contribute to greater excitability within the circuit. We showed previously that activation of nAChRs on glutamatergic terminals can enhance long-term potentiation (LTP) induction at the excitatory inputs to VTA dopamine neurons (Mansvelder and McGehee, 2000). This type of activity-dependent plasticity is induced by even a single exposure to several different drugs of abuse (Saal et al., 2003) and is likely a key element in the acquisition of drug self-administration. Stron- ger $\mathrm{nAChR}$ responses in HR rats, particularly on glutamate afferents and dopamine somata, would be expected to lower the threshold for LTP induction by nicotine and likely other drugs of abuse.

Increased excitability of VTA GABA neurons by stronger $\mathrm{nAChR}$ effects in HR rats would be expected to increase local inhibitory drive to the dopamine neurons. Compared with the modulation of glutamatergic activity and direct excitation of the dopamine neurons by nAChRs, however, the HR/LR differences in GABAergic modulation were smaller in magnitude and prevalence. This is reflected in a smaller difference in percentage of responsive cells in HR versus LR, as well as a smaller $r$ value, reflecting the correlation of response magnitudes with locomotor response to novelty. Thus, we expect that the net effect of the observed increase in nAChR effects will be an enhancement of dopamine neuron excitability and, by extension, greater reactivity of the circuitry to addictive drugs. It is possible that enhancement of nAChR effects on inhibitory interneurons within the VTA may be adaptive for limiting excitability to protect against excitotoxicity.

Because dopamine neurons in the VTA and their synaptic inputs are regulated by endogenous cholinergic afferents, it is reasonable to predict a major role for cholinergic drive in the generation of motivated behaviors. In fact, recent evidence has shown an important role of nAChRs in the motivational properties of psychostimulants, nicotine, ethanol, and intracranial selfstimulation (Yeomans and Baptista, 1997; Picciotto et al., 1998; Soderpalm et al., 2000; Zachariou et al., 2001; Schoffelmeer et al., 2002). Most notably, nAChRs have been implicated in the induction of behavioral and biochemical sensitization. The observation that sensitized midbrain dopamine neurotransmission is associated with enhanced drug self-administration (De Vries et al., 1998; Vezina et al., 2002) suggests that nAChRs may be critical for the development of drug addiction. Because HR animals exhibit sensitized behavioral and biochemical responses and more readily self-administer drugs of abuse, they provide a good model for investigating the inherent factors that underlie drug addiction.

In our in vitro and in vivo assays, glucocorticoid receptors were necessary for stress hormone-related changes in nAChR function. In addition to affecting drug self-administration, sensitization, and relapse, stressful experience has also been shown to influence VTA glutamate receptor expression in a glucocorticoid receptor-dependent manner (Saal et al., 2003). This suggests that many receptor subtypes are affected by stress hormones. Whether enhanced nAChR activity contributes the observed changes in glutamate receptors under stressful conditions, or after exposure to drugs of abuse, remains to be investigated. Ultimately, a combination of cellular properties likely mediate drug abuse vulnerability, and it will be interesting to continue to test their potential importance in establishing the vulnerable phenotype.

In our studies of stress hormone effects, changes in nAChR responses were seen after $48 \mathrm{~h}$ of CORT exposure or stress, which may be relevant to behaviors induced by chronic stress. In human psychophysical studies, subjective perception of novel amphetamine is not affected by acute stress hormone administration (Wachtel et al., 2001). HR animals experience persistent elevations in stress hormones compared with LR animals for a significant portion of their lives (Marinelli and Piazza, 2002). However, antagonist or agonist-induced upregulation of $\mathrm{nAChR}$ function can be seen after just a few hours and reaches a maximum in $<12$ h (Buisson and Bertrand, 2001; Vallejo et al., 2005). Thus, the 
time course and RU486 sensitivity of CORT effects on nAChR function most likely result from changes in gene expression, with minimal contribution of direct interactions of the hormone with nAChRs. In addition, our functional evidence suggests that receptors composed of several nAChR subunits are affected. Given that these subunits are encoded by disparate genes on different chromosomes, identifying the mechanisms linking glucocorticoid receptors to nAChR function and/or expression will be a significant challenge.

This study provides insight into the cellular differences that exist in animals predisposed to self-administer drugs of abuse. Cholinergic drive to the VTA contributes to dopamine neuron activity during normal behavior (Blaha et al., 1996). As such, alterations in nAChR sensitivity would be expected to result in profound effects on the interpretation of rewarding substances and behaviors. This study provides evidence for a potentially important component of the cellular and molecular substrates underlying the inherent vulnerability to drug abuse, as well as a mechanism for establishing vulnerability through chronic environmental stress.

\section{References}

Almeida LE, Pereira EF, Alkondon M, Fawcett WP, Randall WR, Albuquerque EX (2000) The opioid antagonist naltrexone inhibits activity and alters expression of alpha7 and alpha4beta2 nicotinic receptors in hippocampal neurons: implications for smoking cessation programs. Neuropharmacology 39:2740-2755.

Blaha CD, Allen LF, Das S, Inglis WL, Latimer MP, Vincent SR, Winn P (1996) Modulation of dopamine efflux in the nucleus accumbens after cholinergic stimulation of the ventral tegmental area in intact, pedunculopontine tegmental nucleus-lesioned, and laterodorsal tegmental nucleus-lesioned rats. J Neurosci 16:714-722.

Buisson B, Bertrand D (2001) Chronic exposure to nicotine upregulates the human $\alpha 4 \beta 2$ nicotinic acetylcholine receptor function. J Neurosci $21: 1819-1829$.

Choi HK, Won LA, Kontur PJ, Hammond DN, Fox AP, Wainer BH, Hoffmann PC, Heller A (1991) Immortalization of embryonic mesencephalic dopaminergic neurons by somatic cell fusion. Brain Res 552:67-76.

Dani JA (2003) Roles of dopamine signaling in nicotine addiction. Mol Psychiatry 8:255-256.

Deroche V, Marinelli M, Le Moal M, Piazza PV (1997) Glucocorticoids and behavioral effects of psychostimulants. II: Cocaine intravenous selfadministration and reinstatement depend on glucocorticoid levels. J Pharmacol Exp Ther 281:1401-1407.

De Vries TJ, Schoffelmeer AN, Binnekade R, Mulder AH, Vanderschuren LJ (1998) Drug-induced reinstatement of heroin- and cocaine-seeking behaviour following long-term extinction is associated with expression of behavioural sensitization. Eur J Neurosci 10:3565-3571.

Dong K, Qu T, Ahmed FA, Zhang L, Yamada K, Guison NG, Miller M, Yamadori T (1996) Fluoro-Green and Fluoro-Red: two new fluorescent retrograde tracers with a number of unique properties. Brain Res 736:61-67.

Ford CP, Mark GP, Williams JT (2006) Properties and opioid inhibition of mesolimbic dopamine neurons vary according to target location. J Neurosci 26:2788-2797.

Garzón M, Vaughan RA, Uhl GR, Kuhar MJ, Pickel VM (1999) Cholinergic axon terminals in the ventral tegmental area target a subpopulation of neurons expressing low levels of the dopamine transporter. J Comp Neurol 410:197-210.

Hooks MS, Jones GH, Smith AD, Neill DB, Justice JB, Jr (1991) Response to novelty predicts the locomotor and nucleus accumbens dopamine response to cocaine. Synapse 9:121-128.

Klink R, de Kerchove d'Exaerde A, Zoli M, Changeux JP (2001) Molecular and physiological diversity of nicotinic acetylcholine receptors in the midbrain dopaminergic nuclei. J Neurosci 21:1452-1463.

Koob GF, Le Moal M (1997) Drug abuse: hedonic homeostatic dysregulation. Science 278:52-58.

Mansvelder HD, McGehee DS (2000) Long-term potentiation of excitatory inputs to brain reward areas by nicotine. Neuron 27:349-357.

Mansvelder HD, McGehee DS (2002) Cellular and synaptic mechanisms of nicotine addiction. J Neurobiol 53:606-617.
Mansvelder HD, Keath JR, McGehee DS (2002) Synaptic mechanisms underlie nicotine-induced excitability of brain reward areas. Neuron 33:905-919.

Mantsch J, Saphier D, Goeders N (1998) Corticosterone facilitates the acquisition of cocaine self-administration in rats: opposite effects of the type II glucocorticoid receptor agonist dexamethasone. J Pharmacol Exp Ther 287:72-80.

Margolis EB, Lock H, Hjelmstad GO, Fields HL (2006) The ventral tegmental area revisited: is there an electrophysiological marker for dopaminergic neurons? J Physiol (Lond) 577:907-924.

Marinelli M, Piazza PV (2002) Interaction between glucocorticoid hormones, stress and psychostimulant drugs. Eur J Neurosci 16:387-394.

Omelchenko N, Sesack SR (2005) Laterodorsal tegmental projections to identified cell populations in the rat ventral tegmental area. J Comp Neurol 483:217-235.

Omelchenko N, Sesack SR (2006) Cholinergic axons in the rat ventral tegmental area synapse preferentially onto mesoaccumbens dopamine neurons. J Comp Neurol 494:863-875.

Paxinos G, Watson C (1998) The rat brain in stereotaxic coordinates, Ed 4. San Diego: Academic.

Paxinos G, Watson C (2004) The rat brain in stereotaxic coordinates, Ed 4. New York: Elsevier.

Piazza PV, Deminiere JM, Le Moal M, Simon H (1989) Factors that predict individual vulnerability to amphetamine self-administration. Science 245:1511-1513.

Piazza PV, Deroche V, Rouge-Pont F, Le Moal M (1998) Behavioral and biological factors associated with individual vulnerability to psychostimulant abuse. NIDA Res Monogr 169:105-133.

Piazza PV, Maccari S, Deminiere JM, Le Moal M, Mormede P, Simon H (1991) Corticosterone levels determine individual vulnerability to amphetamine self-administration. Proc Natl Acad Sci USA 88:2088-2092.

Piazza PV, Deroche-Gamonent V, Rouge-Pont F, Le Moal M (2000) Vertical shifts in self-administration dose-response functions predict a drugvulnerable phenotype predisposed to addiction. J Neurosci 20:4226-4232.

Picciotto MR, Zoli M, Rimondini R, Lena C, Marubio LM, Pich EM, Fuxe K, Changeux JP (1998) Acetylcholine receptors containing the beta2 subunit are involved in the reinforcing properties of nicotine. Nature 391:173-177.

Pidoplichko VI, DeBiasi M, Williams JT, Dani JA (1997) Nicotine activates and desensitizes midbrain dopamine neurons. Nature 390:401-404.

Rupprecht R, Holsboer F (1999) Neuropsychopharmacological properties of neuroactive steroids. Steroids 64:83-91.

Saal D, Dong Y, Bonci A, Malenka RC (2003) Drugs of abuse and stress trigger a common synaptic adaptation in dopamine neurons. Neuron 37:577-582.

Schoffelmeer AN, De Vries TJ, Wardeh G, van de Ven HW, Vanderschuren LJ (2002) Psychostimulant-induced behavioral sensitization depends on nicotinic receptor activation. J Neurosci 22:3269-3276.

Shi L-J, He Y-Y, Liu L-A, Wang C-A (2001) Rapid non-genomic effect of corticosterone on neuronal nicotinic acetylcholine receptor in PC12 cells. Arch Biochem Biophys 394:145-150.

Soderpalm B, Ericson M, Olausson P, Blomqvist O, Engel JA (2000) Nicotinic mechanisms involved in the dopamine activating and reinforcing properties of ethanol. Behav Brain Res 113:85-96.

Suto N, Austin JD, Vezina P (2001) Locomotor response to novelty predicts a rat's propensity to self-administer nicotine. Psychopharmacology (Berl) 158:175-180.

Vallejo YF, Buisson B, Bertrand D, Green WN (2005) Chronic nicotine exposure upregulates nicotinic receptors by a novel mechanism. J Neurosci 25:5563-5572.

Vezina P, Lorrain DS, Arnold GM, Austin JD, Suto N (2002) Sensitization of midbrain dopamine neuron reactivity promotes the pursuit of amphetamine. J Neurosci 22:4654-4662.

Wachtel SR, Charnot A, de Wit H (2001) Acute hydrocortisone administration does not affect subjective responses to $d$-amphetamine in humans. Psychopharmacology 153:380-388.

Yeomans J, Baptista M (1997) Both nicotinic and muscarinic receptors in ventral tegmental area contribute to brain-stimulation reward. Pharmacol Biochem Behav 57:915-921.

Zachariou V, Caldarone BJ, Weathers-Lowin A, George TP, Elsworth JD, Roth RH, Changeux JP, Picciotto MR (2001) Nicotine receptor inactivation decreases sensitivity to cocaine. Neuropsychopharmacology 24:576-589. 